INPLASY

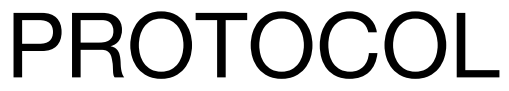

To cite: Liu et al. Efficacy and safety of acupuncture combined with analgesics on pain in Parkinson's patients: A protocol for systematic review and meta-analysis. Inplasy

protocol 202170100. doi:

10.37766/inplasy2021.7.0100

Received: 31 July 2021

Published: 31 July 2021

Corresponding author:

Ruofei Liu

1563824879@qq.com

Author Affiliation:

Jiangxi University of Chinese

Medicine.

Support: Grant No.2013A24.

Review Stage at time of this submission: Preliminary

searches.

Conflicts of interest:

None declared.

\section{Efficacy and safety of acupuncture combined with analgesics on pain in Parkinson's patients: A protocol for systematic review and meta-analysis}

Liu, RF1; Fan, P2; Zhan, QN3; Zhang, Q4; Chen, SQ5; Kuang, RQ6.

Review question / Objective: The aims are: 1. to evaluate the efficacy and safety of acupuncture combined with analgesics in the treatment of pain in Parkinson's patients. 2. to provide the latest evidence of evidence-based medicine for the clinical treatment of pain in Parkinson's patients.

Information sources: The English databases include search Embase, Pubmed, Cochrane Central Controlled Trials Register (Central), Medline, China National Knowledge Infrastructure (CNKI), China Biomedical Literature Database (CBM), Wanfang Database, and Chinese Science Journal Database (VIP database). From the establishment of the database to June, 2021. The key words include "acupuncture", "body acupuncture", "electro-acupuncture", "auricular acupuncture", "analgesics", "Parkinson's disease", "pain". In addition, we will also retrieve ongoing or unpublished trials from the International Clinical Trial Registration Platform and Chinese Clinical Trial Registry Platform.

INPLASY registration number: This protocol was registered with the International Platform of Registered Systematic Review and Meta-Analysis Protocols (INPLASY) on 31 July 2021 and was last updated on 31 July 2021 (registration number INPLASY202170100).

\section{INTRODUCTION}

Review question / Objective: The aims are: 1. to evaluate the efficacy and safety of acupuncture combined with analgesics in the treatment of pain in Parkinson's patients. 2. to provide the latest evidence of evidence-based medicine for the clinical treatment of pain in Parkinson's patients.
Condition being studied: Neurological disorders are now the leading source of disability globally, Parkinson's disease (PD) is currently the fastest growing neurological disorder in the world.(1) According to related reports, by 2040, the number of Parkinson's patients worldwide is expected to exceed 14 million. Smoking, pesticides, heavy metals, etc. are related to 
the production of Parkinson's.According to the clinical manifestations of the disease, Parkinson's disease can be divided into motor symptoms and non-motor symptoms (NMS). Pain is a common NMS, from the prodromal stage to the remission stage, it accounts for $98.6 \%$ of NMS reports. At the same time, a large amount of evidence from imaging and electrophysiological studies shows that the pathophysiological mechanism of pain in Parkinson's patients is derived from the dysfunction of the central and peripheral parts of the nociceptive pathway.(5) For the pain of Parkinson's patients, the main clinical treatment measures currently are the use of analgesics such as apomorphine and oxycodone.However, due to the neurotoxicity and gastrointestinal side effects of analgesics, such as constipation,cause the patient's resistance to analgesics. Acupuncture, physical therapy, and surgery provide more options for Parkinson's pain. As a traditional Chinese medicine, acupuncture has been used clinically for a long time to relieve pain, such as cancer pain and chronic pain. The mechanism of acupuncture for pain relief in Parkinson's patients, on the one hand, due to the increase in connections including primary somatosensory cortex (S1), the middle temporal gyrus (MTG), superior marginal gyrus, and guide lobe cortex, these areas may change the perception of nociceptive receptors to the pain matrix. On the other hand, acupuncture can regulate the human nerve-endocrine-immune system and activate the production and release of endogenous opioids. In addition, studies have shown that acupuncture can regulate the dopaminergic pathway by stimulating the medial orbitofrontal cortex (OFC) and finally relieve the pain at night in $P D$ patients. In recent years, a large amount of relevant research data has shown that acupuncture can relieve pain. Although studies have shown that acupuncture combined with analgesics can significantly alleviate the pain symptoms of Parkinson's patients, there is a lack of relevant comprehensive evaluation. Therefore, we carried out this study to further evaluate the efficacy and safety of acupuncture combined with analgesics in the treatment of pain in Parkinson's patients, and to provide clinical evidence.

\section{METHODS}

Participant or population: All the patients included in the study were diagnosed pain in Parkinson's patients, regardless of age, sex, country, race and course of disease.

Intervention: The treatment plan is acupuncture combined with analgesics. Acupuncture treatment includes all types, such as electro-acupuncture, fire acupuncture, body acupuncture, and so on.

Comparator: The control interventions will be treated with Conventional analgesic treatment.

\section{Study designs to be included: RCT.}

Eligibility criteria: All RCTs of acupuncture combined with analgesics in the treatment of pain in Parkinson's patients, whether blinded or unblinded. Case reports casecontrolled studies will be excluded.

Information sources: The English databases include search Embase, Pubmed, Cochrane Central Controlled Trials Register (Central), Medline, China National Knowledge Infrastructure (CNKI), China Biomedical Literature Database (CBM), Wanfang Database, and Chinese Science Journal Database (VIP database). From the establishment of the database to June, 2021. The key words include "acupuncture", "body acupuncture", "electro-acupuncture", "auricular acupuncture", "analgesics", "Parkinson's disease", "pain". In addition, we will also retrieve ongoing or unpublished trials from the International Clinical Trial Registration Platform and Chinese Clinical Trial Registry Platform.

Main outcome(s): The main outcome measure was the total effective rate of analgesia (excluding the evaluation of inefficiency and non-remissionrate). 
Additional outcome(s): The secondary outcome indicators were the King' s Parkinson's Disease Pain Scale (KPPS), which is the most recently developed scale for evaluating pain in patients with PD and adverse reactions.

Quality assessment / Risk of bias analysis: Two reviewers will use Cochrane collaborative tools to evaluate the quality of the literature(17). It includes the following 7 domains: random sequence generation, allocation concealment, blinding of participants and personnel, blinding of outcome assessment, incomplete outcome data, selective reporting and other sources of bias. Each domain includes a judgment of low, high and unclear risk of bias according to information provided by authors. Disagreements between reviewers will be resolved through discussion with a third reviewer.

Strategy of data synthesis: When calculating the effect size, the relative risk and $95 \%$ confidence intervals are used for the dichotomous outcomes, and the weighted mean difference or standard mean difference will be used for the continuous outcomes. If the studies with no statistical homogeneity, the fixed-effect model can be used for analysis; if the studies with significant statistical heterogeneity, random effects model analysis will be used. The Review Manager (RevMan) V.5.3 software will be used for statistical analysis.

Subgroup analysis: Subgroup analysis will be performed if there have heterogeneity between the study results, following items will be considered: type of acupuncture, gender, age.

Sensitivity analysis: We will eliminate the "high-risk" low-quality articles for sensitivity analysis to judge the robustness of the results.

Country(ies) involved: China.

Keywords: Parkinson's disease; Pain; acupuncture; systematic review; protocol.
Contributions of each author:

Author 1 - Ruofei Liu - Conceptualization Software Writing - original draft Writing review \&editing.

Email: 1563824879@qq.com

Author 2 - Ping Fan - Conceptualization Formal analysis Supervision.

Email: 13707087860@163.com

Author 3 - Qinan Zhan - Data curation Supervision Writing - original draft Methodology.

Email: 271413700@qq.com

Author 4 - Qi Zhang - Data curation Formal analysis.

Email: 835447980@qq.com

Author 5 - Siqi Chen - Data curation Methodology Software Writing - review \& editing.

Email: 254490090@qq.com

Author 6 - Renqing Kuang Conceptualization Writing - original draft.

Email: 1556338577@qq.com. 